\title{
Evaluación de la Enseñanza y Aprendizaje por Parte de Estudiantes de un Programa de Talentos Académicos (BETA- PUCV) Frente a un Curso de Neuroanatomía Funcional
}

\author{
Evaluation of Teaching and Learning by Gifted Students of an \\ Enrichment Program (BETA-PUCV) of a Functional Neuroanatomy Course
}

"Pablo José Lizana Arce; "Atilio Aldo Almagià Flores; **María Cristina Simpson Lelievre; *** Octavio Binvignat Gutiérrez; "Ricardo Andrés Henríquez Flores; ${ }^{* * * *}$ María Paz Gómez Arízaga; ${ }^{* * * * *}$ Marcela González Navarro \& ${ }^{* * * * *}$ María Leonor Conejeros Solar

LIZANA, P. J.; ALMAGIÀ, A. F.; SIMPSON, C.; BINVIGNAT, G. O.; HENRÍQUEZ, R.; GÓMEZ, M.; GONZÁLEZ, M. \& CONEJEROS, L. Evaluación de la enseñanza y aprendizaje por parte de estudiantes de un programa de talentos académicos (BETAPUCV) frente a un curso de neuroanatomía funcional. Int. J. Morphol., 28(4):1245-1249, 2010.

RESUMEN: El talento académico se presenta en aproximadamente un $6 \%$ de la población, y se caracteriza por un destacado desempeño intelectual en algún área específica del conocimiento o en áreas generales. Esta investigación explora la aplicación de un método de enseñanza activo-participativo en el área de la neuroanatomía funcional, a estudiantes con talento académico de cursos de Enseñanza media $(n=27)$, que formaban parte de un programa de enriquecimiento curricular (Programa BETA-PUCV). Este método integra las clases interactivas, la realización de pasos prácticos que incluyen disecciones, la confección de modelos tridimensionales y la preparación de técnicas anatómicas, además de un refuerzo virtual a través del Aula Virtual de Anatomía Humana. Los estudiantes formaron parte de cursos impartidos durante el primer y segundo semestre 2008. Al fin del período, los estudiantes evalúan el curso mediante una encuesta que contiene preguntas en escala Likert y una sección cualitativa. Los resultados de la comparación de las evaluaciones realizadas por los estudiantes que participaron en dichos cursos versus estudiantes de otros cursos del área científica del programa BETA, demuestran que a través de las metodologías utilizadas, se han maximizando las posibilidades de aprendizaje de los estudiantes. Esto, se ha reflejado en diferencias significativas de nuestra propuesta versus el resto de los cursos. El talento académico que presenta este tipo de estudiantes sumado a la metodología de trabajo, permiten que cada estudiante avance a su propio ritmo y además descubra a través de la experiencia directa la morfología del sistema nervioso.

PALABRAS CLAVE: Talentos académicos; Enseñanza y aprendizaje; Neuroanatomía.

\section{INTRODUCCIÓN}

El concepto de talento posee diferentes definiciones y ha variado considerablemente a través del tiempo. Para fines de esta investigación, se ha adoptado la definición de Gagné (2008), quien se refiere a la existencia, aproximadamente en el $10 \%$ de la población, de aptitudes naturales sobresalientes o dotación. Estas aptitudes se pueden presentar en diferentes dominios (e.g. intelectual, creativo, socioafectivo) y, progresivamente, se transforman en habilidades sistemáticamente desarrolladas, o talentos. Para que esto suceda, se deben activar y actuar en conjunto los llamados catalizadores intrapersonales y ambientales. La educación, tanto formal como informal, constituyen las formas más privilegiadas para desarrollar y consolidar las posibilidades que las diferentes dotaciones se expresen en talentos consolidados (Artola et al., 2005). Una de las instituciones que ha pretendido instalarse como uno de los catalizadores ambientales de estudiantes con talento potencial de la $\mathrm{V}$ Región, es el programa BETA-PUCV. BETA es un progra-

\footnotetext{
Laboratorio de Antropología Física y Anatomía Humana, Laboratorio de Técnicas Anatómicas, Instituto de Biología, Facultad de Ciencias, Pontificia Universidad Católica de Valparaíso, Chile.

** Instituto de Estadística, Pontificia Universidad Católica de Valparaíso, Chile.

*** $\quad$ Facultad de Ciencias de la Salud. Universidad Autónoma de Chile.

***** University of Arizona, Department of Disability and Psychoeducational Studies, USA.

***** Programa BETA-PUCV (Buenos Estudiantes con Talentos Académicos - Pontificia Universidad Católica de Valparaíso), Chile.
} 

parte de estudiantes de un programa de talentos académicos (BETA-PUCV) frente a un curso de neuroanatomía funcional. Int. J. Morphol., 28(4):1245-1249, 2010.

ma de enriquecimiento extracurricular para estudiantes con talento potencial, creado el año 2005, en la Pontificia Universidad Católica de Valparaíso y su objetivo general es "construir un contexto educativo que otorgue oportunidades de alta calidad para contribuir al desarrollo integral, comportamientos prosociales y habilidades de orden superior de niños, niñas y jóvenes con talento académico de la Región de Valparaíso, Chile.

En el marco del objetivo general del programa Beta, esto es, otorgar oportunidades educativas para los estudiantes con talento potencial, el área de ciencias básicas, específicamente neurociencias, se ha instalado como un curso estable dentro del programa para que los estudiantes puedan adquirir conocimientos específicos y desarrollar habilidades superiores de pensamiento.

Actualmente, existe información relevante en el campo de la educación formando "puentes" hacia la neurociencia, este término se refiere a derribar obstáculos epistemológicos que propicien la integración de conocimientos (Benarós et al., 2010). Es así, como varios autores han considerando importantes hallazgos de la neurociencia para su aplicación en procesos de enseñanza y aprendizaje (Geake \& Cooper, 2003; Goswami, 2004; Goswami, 2006; Hall, 2005), incluso ha suscitado importantes seminarios internacionales en nuestro país (Hirsh-Pasek \& Bruer, 2007). Sin embargo, aun no existe una propuesta concreta en el ámbito de enseñanza formal y para talentos académicos con el fin de incluir estos claros avances de la neurociencia en la práctica educativa. Actualmente, existen varias indicaciones que plasman el resultado de las investigaciones en neurociencia, aplicadas a la educación (Jensen, 2004), existiendo centros exclusivos para el estudio que interrelaciona neurociencia y educación (Cambridge University, 2009).

El Modelo Neuroeducativo que se propone, es un programa cuyo objetivo es aportar a la construcción del enlace entre neurociencia y educación. Se basa principalmente en los mecanismos generales de aprendizaje y memoria, y su aplicación a estrategias educativas concretas. En este modelo se incluyen las principales variables que influyen en el aprendizaje, las que llamamos factores de aprendizaje, de acuerdo a las investigaciones de neurociencia, las que son implementadas y potenciadas en tres entornos integrados, a los que hemos denominado los Entornos Aula, Práctico y Virtual (Lizana, 2007, Lizana et al., 2008) (Fig. 1).

La presente investigación pretende evaluar el impacto del modelo neuroeducativo en los aprendizajes de los estudiantes, de modo de aportar a un mejoramiento en los cursos de talentos académicos del área de las ciencias morfológicas, a través de un modelo educativo diseñado de acuerdo a una revisión de las prácticas educativas existentes y la incorporación de estrategias pedagógicas fundamentadas en los mecanismos generales de procesamiento del sistema nervioso.

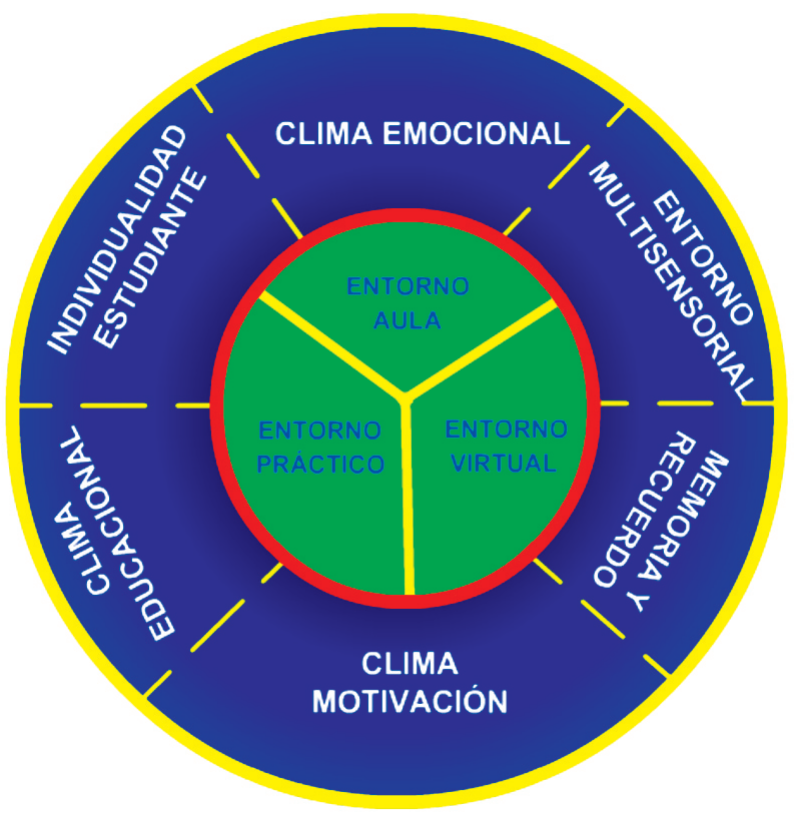

Fig. 1. Diagrama que representa el modelo neuroeducativo. El círculo externo o periférico (en azul) representa cada una de los factores que promueven el aprendizaje que se incluyen en los tres entornos propuestos. Los factores están íntimamente relacionados y por eso, se esquematizan líneas con espacios entre cada factor. El núcleo central del modelo (en verde) está representado por tres entornos que se articulan íntimamente el uno con el otro, que son: entorno aula, entorno práctico y entorno virtual, para favorecer el aprendizaje de los estudiantes. En el medio deben estar los estudiantes (Tomado de Lizana, 2007).

\section{SUJETOS Y MÉTODO}

Los participantes de esta investigación fueron 27 estudiantes, de ambos géneros, de enseñanza media del programa BETA, que cursaron la asignatura "Neurociencia: desde las neuronas al cómo aprendemos", impartida durante el primer y segundo semestres de 2008. El método aplicado durante el curso incluyó las siguientes estrategias: (a) clases interactivas, donde se presentaron y discutieron los temas, se realizaron pasos prácticos (ej. disecciones), (b) confección de modelos tridimensionales, (c) seminarios de investigación y técnicas anatómicas, y (d) un refuerzo continuo a través del Aula Virtual de Anatomía Humana. 
LIZANA, P. J.; ALMAGIÀ, A. F.; SIMPSON, C.; BINVIGNAT, G. O.; HENRÍQUEZ, R.; GÓMEZ, M.; GONZÁLEZ, M. \& CONEJEROS, L. Evaluación de la enseñanza y aprendizaje por parte de estudiantes de un programa de talentos académicos (BETA-PUCV) frente a un curso de neuroanatomía funcional. Int. J. Morphol., 28(4):1245-1249, 2010.

Procedimiento. Para evaluar la percepción de los estudiantes sobre los métodos utilizados se aplicó, al final del curso, un cuestionario con respuestas en escala Likert (i.e. totalmente de acuerdo, de acuerdo, en parte de acuerdo, en desacuerdo), El cuestionario se encontraba dividido en tres secciones: I. Percepciones sobre el curso; II. Percepciones sobre el profesor III. Autoevaluación del estudiante. Estas encuestas son de elaboración del Programa BETA-PUCV, las que fueron aplicadas, por una persona externa al curso y sin la presencia de los docentes en el aula.

Análisis. Los resultados del curso "Neurociencia: desde las neuronas al cómo aprendemos" fueron comparados-mediante el uso de pruebas T para grupos independientes - con otro curso de ciencias (grupo control) realizado en el programa BETA para estudiantes de Enseñanza Media, en el mismo período $(\mathrm{n}=35)$. Además, se contrastaron los resultados del curso con el resto de los cursos del programa BETA ( $n=27$ contrastado con $n=296$ en el mismo período) mediante el test Ji-cuadrado.

\section{RESULTADOS}

En las Tablas I, II y III se presentan los análisis comparativos realizados frente a las preguntas que conforman las tres secciones de la encuesta (comparación estudiantes de neurociencia versus resto de los cursos BETA y con el curso de ciencia). Cabe mencionar que dado que dichas encuestas tuvieron variaciones, se escogieron sólo los enunciados que coincidían entre los años estudiados.

Tabla I. Evaluación de la percepción de los estudiantes sobre el curso "Neurociencia: desde las neuronas al cómo aprendemos".

\begin{tabular}{lcccc}
\hline Afirmación & $\begin{array}{c}\text { Curso } \\
\text { Neurociencia }\end{array}$ & $\begin{array}{c}\text { Resto de } \\
\text { cursos BETA }\end{array}$ & $\begin{array}{c}\text { Curso Neurociencia/resto } \\
\text { de cursos Beta Valor-p }\end{array}$ & $\begin{array}{c}\text { Curso Neurociencia/Curso } \\
\text { ciencia Beta Valor-p }\end{array}$ \\
\hline $\begin{array}{l}\text { Los contenidos abordados en el curso } \\
\text { taller te resultaron interesantes. }\end{array}$ & $96,3 \%^{*}$ & $67,2 \%$ & $0,002 *$ & No comparado \\
$\begin{array}{l}\text { Sientes que en este curso/taller } \\
\text { aprendiste mucho. }\end{array}$ & $96,3 \% *$ & $62,8 \%$ & $0,000^{*}$ & $0,000^{*}$ \\
\hline
\end{tabular}

(*) Indica diferencias significativas $\mathrm{p}<0,05$.

Tabla II. Evaluación de la percepción de los estudiantes sobre el profesor.

\begin{tabular}{|c|c|c|c|c|}
\hline Afirmación & $\begin{array}{c}\text { Curso } \\
\text { Neurociencia }\end{array}$ & $\begin{array}{c}\text { Resto de } \\
\text { cursos } \\
\text { BETA }\end{array}$ & $\begin{array}{l}\text { Curso Neurociencia/ } \\
\text { resto de cursos Beta } \\
\text { Valor-p }\end{array}$ & $\begin{array}{c}\text { Curso Neurociencia } \\
\text { Curso ciencia Beta } \\
\text { Valor-p }\end{array}$ \\
\hline Te gustó la manera de enseñar del profesor. & $96,3 \% *$ & $65,9 \%$ & $0,001 *$ & $0,004 *$ \\
\hline Te hizo pensar o descubrir cosas nuevas & $96,3 \% *$ & $67,2 \%$ & $0,002 *$ & $0,024 *$ \\
\hline Utilizó distintas formas de trabajo en clases & $100 \% *$ & $58,4 \%$ & $0,000^{*}$ & $0,000^{*}$ \\
\hline Se mostró dis ponib le a responder preguntas & $100 \% *$ & $79,4 \%$ & $0,009 *$ & $0,076 \mathrm{NS}$ \\
\hline Era cercano y te daba confianza & $96,3 \% *$ & $67,2 \%$ & $0,002 *$ & $0,000^{*}$ \\
\hline Era puntual para iniciar y termin ar las clases & $100 \% *$ & $58,1 \%$ & $0,000^{*}$ & $0,000^{*}$ \\
\hline Daba espacios de participación de todos los estudiantes & $96,3 \% *$ & $74,3 \%$ & $0,01^{*}$ & $0,471 \mathrm{NS}$ \\
\hline Ofreció desafíos y propuso metas altas para el aprendizaje de todos & $96,3 \% *$ & $71,6 \%$ & $0,005^{*}$ & $0,292 \mathrm{NS}$ \\
\hline Tomarías otro curso con este profesor & $100 \% *$ & $59,1 \%$ & $0.000^{*}$ & $0.000 *$ \\
\hline
\end{tabular}

(*) Indica diferencias $\mathrm{p}<0,05$. (NS) no significativo.

Tabla III. Autoevaluación del estudiante sobre su desempeño en los cursos.

\begin{tabular}{lccc}
\hline Afirmación & $\begin{array}{c}\text { Curso } \\
\text { Neurociencia }\end{array}$ & $\begin{array}{c}\text { Resto de } \\
\text { cursos BETA }\end{array}$ & $\begin{array}{c}\text { Curso } \\
\text { Neurociencia/resto de } \\
\text { cursos Beta }\end{array}$ \\
\hline $\begin{array}{l}\text { Valor-p } \\
\text { Puse mi mayor esfuerzo en esta clase }\end{array}$ & $63 \%$ & $38,5 \%$ & $0,013 *$ \\
Puse atención al profesor en esta clase & $77,8 \%$ & $59,1 \%$ & $0,059 \mathrm{NS}$ \\
Participé activamente en clases & $51,9 \%$ & $42,2 \%$ & $0,131 \mathrm{NS}$ \\
Colaboré activamente en el apre ndi zaje de mis compañeros & $48,1 \%$ & $38,2 \%$ & $0,005 *$ \\
Profundicé más allá de lo que la clase me pedía & $40,7 \%$ & $28,0 \%$ & $0,741 \mathrm{NS}$ \\
\hline
\end{tabular}

(*) Indica diferencias $\mathrm{p}<0,05$. (NS) no significativo. 
LIZANA, P. J.; ALMAGIÀ, A. F.; SIMPSON, C.; BINVIGNAT, G. O.; HENRÍQUEZ, R.; GÓMEZ, M.; GONZÁLEZ, M. \& CONEJEROS, L. Evaluación de la enseñanza y aprendizaje por parte de estudiantes de un programa de talentos académicos (BETA-PUCV) frente a un curso de neuroanatomía funcional. Int. J. Morphol., 28(4):1245-1249, 2010.

\section{DISCUSIÓN}

Este estudio muestra variantes del aprendizaje anatómico en el área de la neuroanatomía funcional a través de la implementación de un modelo de trabajo basado en las interacciones entre neurociencia y educación (Lizana; Lizana et al., 2008). Esta metodología de trabajo fue implementada y evaluada por estudiantes con talento académico en los ítem de: a) sobre el curso, b) sobre el profesor y c) sobre sí mismos (autoevaluación). Las encuestas realizadas a los estudiantes se centraron en preguntas puntuales donde los estudiantes se sintieron satisfechos en la forma de abordar los contenidos frente a la modalidad propuesta de enseñanza y aprendizaje (Tablas I y II) con porcentajes de aceptación sobre $96,3 \%$, a totalmente satisfechos (100\%). Los resultados de esta implementación avalarían su utilización debido a que en los ítemes evaluados sobre el curso y del profesor las diferencias fueron significativas $(p>0,05)$ con respecto al resto de los cursos BETA, como también en comparación el curso control de ciencias. Estos buenos resultados han sido replicados en otros grupos etarios de estudiantes talentosos de Enseñanza Básica (Lizana et al., 2009), como también en estudiantes de Enseñanza Media (no pertenecientes a un programa de enriquecimiento curricular), donde hubo un mejoramiento en sus aprendizajes medido en calificaciones (Lizana).

Las características del profesor para estudiantes talentosos y para el programa de enriquecimiento curricular también es importante para la toma de decisiones en cuanto a continuidad de cursos. Para esto, el programa establece la promoción de nuevos desafíos metas altas, suscitar el descubrimiento, creatividad y el pensamiento crítico. En este punto también los estudiantes estuvieron de acuerdo con el trabajo docente y la metodología utilizada (Tablas I, II). En relación a esto varios investigadores han descrito las características de los docentes que trabajan con talentos académicos para potenciar la dotación de los estudiantes (Benavides et al., 2004; Cheung \& Phillipson, 2008). La optimización del trabajo y la capacitación para trabajar con este tipo de estudiantes también se ha debido al apoyo profesional brindado por el programa BETA-PUCV.

En cuanto al ítem autoevaluación del estudiante, en su mayoría las diferencias no alcanzan significancia, tanto en la comparación con el curso de ciencias como con el resto de los cursos BETA, además de obtenerse los más bajos porcentajes, esta tendencia la asociamos a su inherente capacidad de autocrítica, al compararlo con estudiantes creativos y de alto rendimiento (Kingore, 2003) y alto grado de perfeccionismo (Schuler, 2000), a pesar que todos los estudiantes lograron los objetivos de aprendizaje planteados en el curso y obteniendo evaluaciones sobresalientes.

En relación a las características socioafectivas de este tipo de estudiantes, vemos que se han identificado su alta sensibilidad e intensidad emotiva (Alencar, 2007). Este punto se visualiza en la pregunta "Era cercano y te daba confianza", donde los estudiantes valoran los aspectos emocionales, lo que está explícitamente contemplado en nuestro modelo de trabajo (clima emocional e individualidad del estudiante), esta planificación de nuestras actividades.

Esta modalidad de trabajo (Lizana; Lizana et al., 2008), combinado con el talento académico que presentan y su avidez de conocimiento, permiten que cada estudiante avance a su propio ritmo y además, descubra a través de la experiencia directa la morfología humana en el área de la neuroanatomía funcional.

AGRADECIMIENTOS. Por el apoyo y financiamiento, a la Dirección de Investigación, Vice-rectoría de Investigación y Estudios Avanzados, Instituto de Biología de la Facultad de Ciencias, a la Dirección de Desarrollo Curricular y Formativo y al programa de enriquecimiento curricular BETA-PUCV. Pontificia Universidad Católica de Valparaíso, Valparaíso, Chile.

LIZANA, P. J.; ALMAGIÀ, A. F.; SIMPSON, C.; BINVIGNAT, G. O.; HENRÍQUEZ, R.; GÓMEZ, M.; GONZÁLEZ, M. \& CONEJEROS, L. Evaluation of teaching and learning by gifted students of an enrichment program (BETA-PUCV) of a functional neuroanatomy course. Int. J. Morphol., 28(4):1245-1249, 2010.

SUMMARY: Academic talent occurs in approximately $6 \%$ of the population and can be defined by a superior intellectual performance in specific or general academic fields. This research explores the application of an active and participative teaching model in the field of functional neuroanatomy to high school academically talented students $(n=27)$ that participate in an enrichment program (BETA program). This method integrates interactive lessons, performance of practical steps that include dissections, constructing tridimensional models and the preparation of anatomic techniques. Students were also reinforced through an internet-based virtual classroom called "Aula Virtual de Anatomía Humana". Students were part of the courses implemented during the program's first and second semester of 2008. At the end of the course students do an evaluation using a survey that includes questions using a Likert scale and a qualitative (open) section. The results of the comparative analysis between students that participated in this course versus students from different science courses of the Beta program show that student learning opportunities have been maximized. This is reflected on the significant differences that support our method. Student academic talent plus the teaching methodology allows each student to progress according to their own rhythm and to discover, through direct experience, the morphology of the nervous system.

KEY WORDS: Academic talent; Teaching and Learning; Neuroanatomy. 
LIZANA, P. J.; ALMAGIÀ, A. F.; SIMPSON, C.; BINVIGNAT, G. O.; HENRÍQUEZ, R.; GÓMEZ, M.; GONZÁLEZ, M. \& CONEJEROS, L. Evaluación de la enseñanza y aprendizaje por parte de estudiantes de un programa de talentos académicos (BETA-PUCV) frente a un curso de neuroanatomía funcional. Int. J. Morphol., 28(4):1245-1249, 2010.

\section{REFERENCIAS BIBLIOGRÁFICAS}

Alencar, E. Características sócio-emocionais do superdotado: questões atuais. Psicol. Estud., 12:371-8, 2007.

Artola, T.; Barraca, M. \& Misterio, P. Niños con altas capacidades: Quiénes son y cómo tratarlo. Madrid, Entha ediciones, 2005.

Benarós, S.; Lipina, S. J.; Segretin, M. S.; Hermida, M. J. \& Jorge, J. A. Neuroscience and education: Towards the construction of interactive bridges. Rev. Neurol., 50:179$86,2010$.

Benavides, M.; Maz, A.; Castro, E. \& Blanco, R. La educación de niños con talento en Iberoamérica. Santiago, OREALC/Unesco, 2004.

Cambridge University. The centre for neuroscience in education, 2010. Disponible en: http:// www.educ.cam.ac.uk/neuroscience/index.htm

Cheung, H. Y. \& Phillipson, S. N. Teachers of gifted students in Hong Kong: Competencies and charateristics. The Asia-Pacific Education Researcher, 17:143-56, 2008.

Gagné, F. Construyendo el talento a partir de la dotación: Breve revisión del MDDT 2.01. Profesor Honorario de Psicología Universidad de Québec, Montréal (Canadá), 2008.

Geake, J. \& Cooper, P. Cognitive Neuroscience: Implications for education?. Westminster Studies in Education, 26:720, 2003.

Goswami, U. Neuroscience and education. Br. J. Educ. Psychol., 74:1-14, 2004

Goswami, U. Neuroscience and education: From research to practice? Nat. Rev. Neurosci., 7:406-13, 2006.

Hall, J. Neuroscience and Education, A review of the contribution of brain science to teaching and learning. SCRE Research, 121:1-35, 2005.

Hirsh-Pasek, K \& Bruer, J. T. The Brain/Education Barrie. Science, 317:1293, 2007.

Jensen, E. Cerebro y aprendizaje, competencias $e$ implicaciones educativas. Madrid, Nancea Ediciones, 2004.
Kingore, B. High achiever, gifted learner, creative thinker. Understanding our gifted, 15:3-5, 2003.

Lizana, P. Diseño y evaluación de un modelo neuroeducativo en cursos de Enseñanza Media. Tesis para optar al Título de Profesor en Biología y Ciencias Naturales, Pontificia Universidad Católica de Valparaíso, 2007.

Lizana, P.; Almagià, A.; Barraza, F. \& Rodríguez, F. Diseño de un modelo neuroeducativo para la enseñanza y aprendizaje de las ciencias morfológicas. Int. J. Morphol., 26:170-226, 2008.

Lizana, P.; Almagià, A.; Binvignat, O.; Henríquez, R.; Arenas, A.; Gómez, M. \& Conejeros, L. Enseñanza y aprendizaje de la morfología del sistema nervioso en estudiantes de un programa de talentos académicos. Int. J. Morphol., 27:245-78, 2009.

Schuler, P. Perfectionsim and gifted adolescents. The Journal of Secondary Gifted Education, 11:183-96, 2000.

Dirección para correspondencia:

Pablo José Lizana Arce

Laboratorio de Antropología Física y Anatomía Humana

Facultad de Ciencias

Pontificia Universidad Católica de Valparaíso

Valparaíso

CHILE

Email: pablo.lizana@ucv.cl

Recibido : 13-09-2010

Aceptado: 22-10-2010 
